\title{
Analysis of a metal clad waveguide sensor having metamaterial as a guiding layer
}

\author{
A. UPADHYAY ${ }^{1}$, Y.K. PRAJAPATI ${ }^{*}$, R. TRIPATHI ${ }^{1}$, V. SINGH ${ }^{2}$, and J.P. SAINI ${ }^{3}$ \\ ${ }^{1}$ Department of Electronics and Communication Engineering, MNNIT, Allahabad (U.P.), India \\ ${ }^{2}$ Department of Physics, Faculty of Science, Banaras Hindu University, Varanasi (U.P.), India \\ ${ }^{3}$ J.P. Saini, Department of Electronics and Communication Engineering, B.I.E.T Jhansi, (U.P.), India
}

\begin{abstract}
In this study a metal clad waveguide sensor with a metamaterial guiding layer is analyzed. Sensitivity of the proposed sensor is derived using dispersion and Fresenal's equations for waveguiding mode and reflection mode. While efficiently analyzing and comparing the results with the existing one, some interesting findings are achieved. It is observed that the proposed sensor shows larger cover layer sensitivity and larger adlayer sensitivity compared to the dielectric guiding layer sensor due to adsorbtive properties of metamaterial. Henceforth, it concludes that the proposed sensor shows sensitivity improvement over a dielectric guiding layer sensor.
\end{abstract}

Keywords: metamaterials, sensitivity, metal clad waveguides, reflection mode, waveguiding mode.

\section{Introduction}

Optical waveguide sensors are prime researcher's attention in recent years due to faster response time, accuracy of specific analyte detection, resistant to electromagnetic interference, compact size and low production cost. First planar waveguide used in gas and humidity sensing was demonstrated by Tiefenthalar [1].The waveguide sensors are also applicable in the field of biosensing [2], chemical sensing [3] and biochemical sensing [4]. Evanescent field waveguide sensors, e.g., planar waveguide sensors are used for the purpose of detecting lipids bilayer, protein adsorption and affinity binding because evanescent fields in these sensors are limited to the range of few hundreds of nanometers [5]. Sensitivity is influenced by ingredient material, construction of a waveguide sensor and analyte on a cover layer. Horvath et al. [6] demonstrated a reverse symmetric waveguide sensor in which refractive index of the cover layer is larger than that of the substrate layer. Because of its structure, evanescent field of the reverse symmetric waveguide penetrates well in a micrometer range the cover layer at cut off film thickness, which is sufficient for the detection of a receptor-legend interaction [7], bacteria monitoring, as well as cell detection $[5,8]$. Metal clad waveguide sensor in which a metal layer is introduced in between substrate and guiding layer can also be used to detect micron scale objects at cut off guiding layer thickness [9].

\footnotetext{
*e-mail: yogendrapra@mnnit.ac.in
}

However, in 1968 a material called metamaterial which is artificially engineered and possessed some unusual behaviour was suggested by Vesselego [10]. In 2000 Pendry reported that metamaterial can enhance the evanescent wave [11]. In 2001 Shelby et al. [12] demonstrated that metamaterial exhibits negative refractive index and forms a left hand rule combination of electric field, magnetic field and wave propagation vector. In 2004 Qing and Cheng verified that metamaterial enhances evanescent field for both TE and TM polarization of incident light [13]. M. Huang in his literature verified that sensitivity of microwave sensors enhanced by using metamaterial filled waveguide sensors [14]. Sub-wavelength imaging is also possible by enhancing the resolution limit using stacked metamaterial structures [15]. Prajapati et al. [16] in his literature verified that metamaterial could be able to enhance the sensitivity of a SPR sensor through four- and five-layer structure by increasing dip in the reflectance curve in TM mode. Sharp peaks are obtained by using metamaterial in a metal clad peak type structure which is useful in sensing application [17]. In this communication, we investigate the sensitivities of the proposed metal clad waveguide sensor in the presence of a guiding layer of metamaterial at the waveguide mode, as well as in the reflection mode. A homogenous adsorption of analyte over guiding layer forms a layer of the thickness $d_{A}$. Thus, the proposed waveguide sensor turned into a five-layer sensor. Effect of the metamaterial guiding layer on a cover layer, as well as adlayer sensitivities are also analysed. The paper is orga- 
nized as follows: Section 2 bears fundamental background and necessary formulas of the proposed sensor. In Sect. 3 the obtained results are analysed and compared with the existing one. Section 4 contains conclusion.

\section{Theoretical background}

The proposed structure of a metal clad waveguide comprises four layers as shown in Fig. 1(a). The proposed sensor bears a metamaterial guiding layer of the refractive index value equals $n_{G}$ and the thickness $d_{G}$ with permittivity and permeability as

$$
\begin{gathered}
\varepsilon_{G}(\omega)=1-\left(\frac{\omega_{p l}^{\prime 2}}{\omega^{2}+i \times \psi \times \omega}\right), \\
\mu_{G}(\omega)=1-\left(\frac{l \times \omega_{p l}^{\prime 2}}{\omega^{2}-\omega_{r s o}^{2}+i \times \psi \times \omega}\right) .
\end{gathered}
$$

Where $\omega_{p l}^{\prime 2}$ is the plasma frequency, $\psi$ is the electron scattering rate, $\omega_{r s o}$ is the resonance frequency and $l$ is the fractional area of unit cell occupied by the split ring and $\omega$ is the used frequency.

Remaining layers of the proposed structure are semi-infinite substrate layer of the refractive index $n_{S}$, metal layer of complex the refractive index $n_{M}$ and the thickness $d_{M}$ and semi-infinite cover layer with the refractive index $n_{C}$. When a waveguide sensor is used as a biosensor, usually a homogenous adsorption of analyte particles occurs over the guiding layer of few nanometer thickness, termed as adlayer. With inclusion of an adlayer, the waveguide sensor is converted into a five-layered sensor. In order to excite the waveguiding mode, it is compulsory to have a refractive index of metamaterial lager compared to surrounding dielectric and metallic media. In this literature metamaterials are assumed to be homogenous and isotropic. To avail the functionality of a waveguide sensor, it is customary to solve the Maxwell equations of a four-layered structure. The obtained solution of Maxwell equations is Helmholtz equation and the solution of Helmholtz equation for each layer results into the following equation

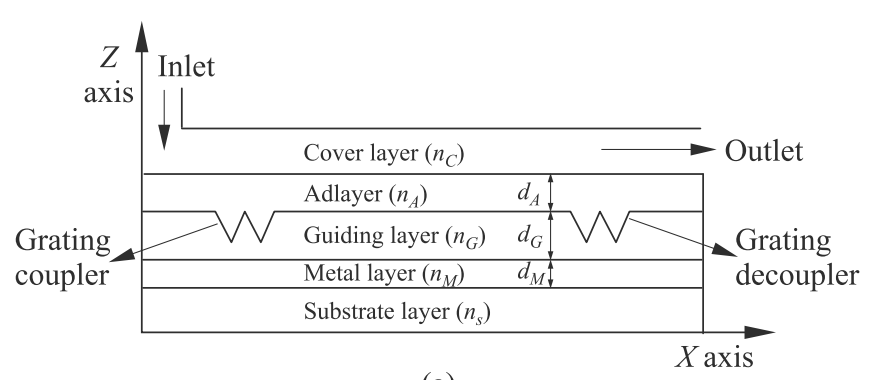

(a)

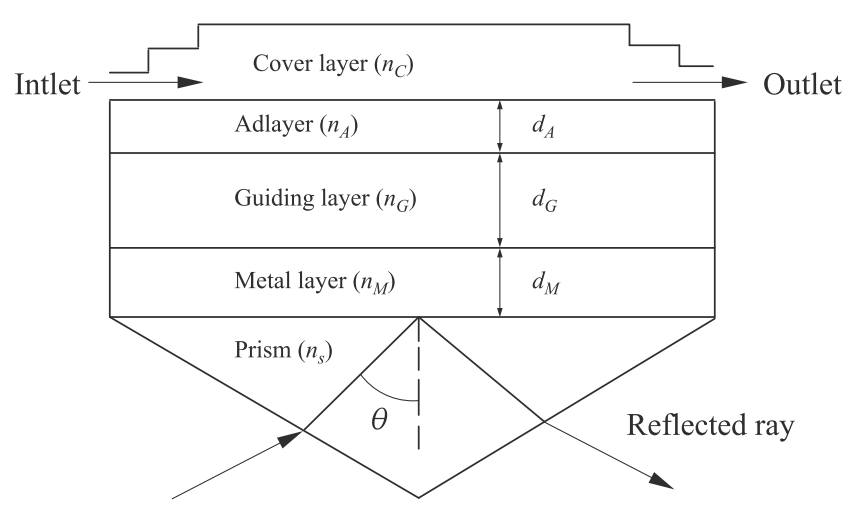

(b)

Fig.1. Schematic diagram of proposed metal clad waveguide sensor with an adlayer having refractive index $n_{A}$ and width $d_{A}$. The refractive indices used are: (a) $n_{S}=1.517, n_{M}=0.065+\mathrm{i} \times 4(\mathrm{Ag})$, $0.15+\mathrm{i} \times 3.2(A u), n_{G}=-\sqrt{[(-4.0+i \times 0.001) \times(-2.4+i \times 0.001)]}$ [25], $n_{A}=1.50, n_{C}=1.33$ or 1.40 (Waveguide mode), (b) $n_{S}=1.517$, $n_{G}=-\sqrt{[(-4.0+i \times 0.001) \times(-3.0+i \times 0.001)]}$ [24] (TE mode), $n_{M}=0.065+\mathrm{i} \times 4(\mathrm{Ag}), 0.15+\mathrm{i} \times 3.2(\mathrm{Au}), n_{A}=1.50, n_{C}=1.33$ (Reflection mode).

intially. Boundary condition for TE mode is $\gamma(z)$ and its derivatives $[d \gamma(d z)] / d z$ are continuous across the boundary. Six equations are obtained for TE mode because there are six values of amplitude in a four-layer waveguide Helmholtz equation solution. These six equations can be constituted as

$$
A \gamma(z)=0
$$

$$
\gamma_{Z}= \begin{cases}{\left[A_{C}^{+} \exp -i\left(k_{X} x+k_{C} z\right)\right] \exp i(w t)} & \text { Cover Layer } \\ {\left[A_{G}^{+} \exp -i\left(k_{X} x+k_{G} z\right)+A_{G}^{-} \exp -i\left(k_{X} x-k_{G} z\right)\right] \exp i(w t)} & \text { Guiding Layer } \\ {\left[A_{M}^{+} \exp -i\left(k_{X} x+k_{M} z\right)+A_{M}^{-} \exp -i\left(k_{X} x-k_{M} z\right)\right] \exp i(w t)} & \text { Metal Layer } \\ {\left[A_{S}^{-} \exp -i\left(k_{X} x-k_{S} z\right)\right] \exp i(w t)} & \text { Substrate Layer }\end{cases}
$$

where $\gamma(z)=\left\{A_{S}^{-}, A_{M}^{+}, A_{M}^{-}, A_{G}^{+}, A_{G}^{-}, A_{C}^{+}\right\}$and $A$ can be represented as 


$$
A=\left[\begin{array}{cccccc}
1 & -1 & -1 & 0 & 0 & 0 \\
-k_{S} & -k_{M} & k_{M} & 0 & 0 & 0 \\
0 & \exp \left(i k_{M} d_{M}\right) & \exp \left(-i k_{M} d_{M}\right) & -\exp \left(i k_{G} d_{M}\right) & -\exp \left(-i k_{G} d_{M}\right) & 0 \\
0 & k_{M} \exp \left(i k_{M} d_{M}\right) & -k_{M} \exp \left(-i k_{M} d_{M}\right) & -k_{G} \exp \left(i k_{G} d_{M}\right) & -k_{G} \exp \left(-i k_{G} d_{M}\right) & 0 \\
0 & 0 & 0 & \exp \left[i k_{G}\left(d_{M}+d_{F}\right)\right] & \exp \left[-i k_{G}\left(d_{M}+d_{F}\right)\right] & \exp \left[-i k_{C}\left(d_{M}+d_{F}\right)\right] \\
0 & 0 & 0 & k_{G} \exp \left[i k_{G}\left(d_{M}+d_{F}\right)\right] & -k_{G} \exp \left[-i k_{G}\left(d_{M}+d_{F}\right)\right]-k_{C} \exp \left[-i k_{C}\left(d_{M}+d_{F}\right)\right]
\end{array}\right]
$$

Here $d_{M}$ and $d_{G}$ are the thickness of metal layer and guiding layer, respectively.

In order to get nontrivial solution of Eq. (4), its determinant must be equal to zero, resultant of which gives the dispersion relation of the proposed waveguide sensor. The equations are as follows

$$
\Phi_{\text {total }}=2 \times k_{G} \times d_{G}+\Phi_{G C}+\Phi_{G M S}-2 \times m \times \pi .
$$

Where

$$
\begin{gathered}
k_{G}=k_{0} \times \sqrt{n_{G}^{2}-n_{\text {eff }}^{2}} \\
\Phi_{G C}=2 \times \tan ^{-1}\left(i \times \frac{k_{C}}{k_{G}}\right), \\
\Phi_{G M S}=\tan ^{-1}\left\{i \times \frac{1-r_{G M}}{1+r_{G M}} \times \frac{1-r_{M S} \times \exp \left(2 \times k_{M} \times d_{M}\right)}{1+r_{M S} \times \exp \left(2 \times k_{M} \times d_{M}\right)}\right\} \\
r_{G M}=\frac{\left(k_{G} / n_{G}^{2 \alpha}\right)-\left(k_{M} / n_{M}^{2 \alpha}\right)}{\left(k_{G} / n_{G}^{2 \alpha}\right)+\left(k_{M} / n_{M}^{2 \alpha}\right)} \\
r_{M S}=\frac{\left(k_{M} / n_{M}^{2 \alpha}\right)-\left(k_{S} / n_{S}^{2 \alpha}\right)}{\left(k_{M} / n_{M}^{2 \alpha}\right)+\left(k_{S} / n_{S}^{2 \alpha}\right)}, \\
k_{J}=k_{0} \sqrt{n_{e f f}^{2}-n_{J}^{2}} .
\end{gathered}
$$

Here $n_{\text {eff }}$ is the effective refractive index of a guiding layer of the metamaterial based waveguide sensor and $n_{\text {eff }}$ can be calculated by using the following formula: $n_{\text {eff }}=n_{G} \times \sin \theta_{G}$ where $\theta_{G}$ is the incident angle at guiding layer medium, $n_{G}$ is the refractive index of guiding layer, $n_{J}$ denotes the refractive index of cover layer/metal layer/substrate layer, $k_{0}$ is the propagation constant of wave in vacuum which can be described as $2 \pi / \lambda$ where $\lambda$ is the used wavelength, while phase shift at interface of guiding layer and cover layer is denoted by $\Phi_{G C}$ and $\Phi_{G M S}$ describes the phase shift at guiding layer-metal layer-substrate layer interface, $d_{G}$ is the guiding layer width, $d_{M}$ is the metal layer width. $m$ signifies the mode number of propagating wave in a waveguide sensor, $r_{M S}$ and $r_{G M}$ are the reflection coefficients at metal layer-substrate layer interface and guiding layer-metal layer interface, respectively and $\alpha=0.1$ for TE and TM mode, respectively [1]. The width of metal layer is $30-40 \mathrm{~nm}$ which is able to make wave propagating mode independent of substrate layer field in a guiding layer [9]. This turns the four-layer waveguide into a three-layer waveguide with metal substrate. Due to the highly reflecting surface and lossy nature of metal layer, electromagnetic waves are highly attenuated in guiding layer region after hundreds of nanometer guiding layer thickness and the output intensity of light is hardly measurable. Therefore, waveguiding mode cannot be used for larger guiding layer thickness. Thus, most of the time metal clad waveguide sensor is used in a reflection mode. In this mode [Fig. 1(b)], the procedure of the operation is to appraise the reflection strength as a mapping of incident angle. In the reflectance mode of a waveguide sensor, the tangential component of an incident wave vector at a prism-metal layer interface corresponds to a wave number of the guided mode allowing the wave to couple with and propagate. Hence, at output in the reflectance curve minima occurs. This minimum point in a reflectance curve forms a dip at a particular angle called waveguide mode angle. From Fresenal's law the reflection equation of the four-layer can be written as

$$
R_{S M G C}=\left|\frac{r_{S M}+r_{M G C} \times \exp \left(2 \times i \times k_{M} \times d_{M}\right)}{1+r_{S M} \times r_{M G C} \times \exp \left(2 \times i \times k_{M} \times d_{M}\right)}\right|^{2},
$$

where $r_{S M}$ and $r_{M G C}$ are the reflection cofficients at a substrate-metal interface and metal-guiding-cover layer interface, respectively and $R$ denotes the reflectance measured as a function of an incident angle denotes Angle (degrees) in the manuscript's figures. These cofficients can be formulated as

$$
\begin{gathered}
r_{S M}=\frac{\left(k_{S} / n_{S}^{2 \alpha}\right)-\left(k_{M} / n_{M}^{2 \alpha}\right)}{\left(k_{S} / n_{S}^{2 \alpha}\right)+\left(k_{M} / n_{M}^{2 \alpha}\right)}, \\
r_{M G C}=\frac{r_{M G}+r_{G C} \times \exp \left(2 \times i \times k_{G} \times d_{G}\right)}{1+r_{M G} \times r_{G C} \times \exp \left(2 \times i \times k_{G} \times d_{G}\right)},
\end{gathered}
$$

where $\alpha=0.1$ for TE and TM modes, respectively while $r_{M G}$ and $r_{G C}$ are the reflection cofficients at metal-guiding layer interface and guiding-cover layer interface, respectively.

Similarly, Fresenal's equation for a five-layer can be written. Hence, using the above equations information about waveguiding mode and reflectace mode of metal clad waveguide can be extracted. 


\section{Result and discussion}

The proposed structure is supposed to be enlightened with an He-Ne laser beam having $\lambda$ that equals $632.8 \mathrm{~nm}$. At this wavelength, it is supposed that metamaterial as a guiding layer (film) of the thickness $d_{G}$ bears electrical permettivity and permeability $\varepsilon_{G}=\varepsilon_{G}^{\prime}+\varepsilon_{G}^{\prime \prime}, \mu_{G}=\mu_{G}^{\prime}+\mu_{G}^{\prime \prime}$, respectively where $\varepsilon_{G}^{\prime}, \mu_{G}^{\prime}$ is the real part and $\varepsilon_{G}^{\prime \prime}, \mu_{G}^{\prime \prime}$ is an imaginary part of permittivity and permeability, respectively. Whereas, the metal layer is assumed to be silver $(A g)$ and gold $(A u)$ having the electrical permittivity $\varepsilon_{M}$ equals $(-16+1 \mathrm{i} \times 0.52)$ and $(-10.22+1 \mathrm{i} \times 0.96)$, respectively while the magnetic permeability $\mu_{M}$ is 1 for both types of metal layer cladding. Glass is taken as a substrate material with the permettivity of $\varepsilon_{S}=2.3$ and the permeability of $\mu_{S}=1$ (BK-7 Prism), adlayer of the thickness $d_{A}$ with the permittivity of $\varepsilon_{A}=2.25$, with the permeability of $\mu_{A}=1$ (in case of assumption that there is homogenous adsorption over film layer, can be considered as thin layer, i.e., a five-layer model) [6] and the analyte medium is assumed to be water with $\varepsilon_{C}=1.77 \& 1.96$ (added impurity in water) and $\mu_{C}=1$. Refractive index of any layer can be calculated as of $n=\sqrt{\varepsilon \times \mu}$. Metal clad using metamaterial as a guiding layer inclusion is examined in waveguiding, as well as a reflection mode in order to analyze the effects of unconventional structure.

Figures 2(a)-2(b) are graphs of penetration depth of an evanescent wave in the cover layer $v s$. metamaterial film thickness. According to figures it is identified that in a waveguiding mode, penetration depth is infinite in cover medium at cut off guiding layer thickness for both the proposed and reference sensor, however, cut off thickness for the proposed sensor is achieved at lower values of metamaterial guiding layer thickness compared to a metal clad sensor, [9] due to the fundamental backward mode [18] of metamaterial guiding layer as shown in Table 1. Hence, the proposed sensor will be of compact size and large biological cells like bacteria and protein adsorption, could be effectively analyzed due to infinite penetration depth of evanescent field at cut off thickness using the proposed sensor [6].

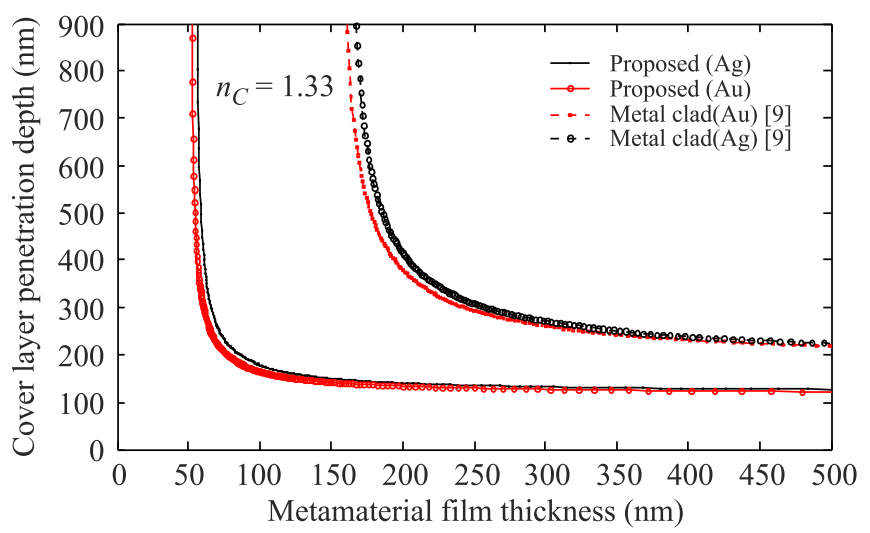

(a)

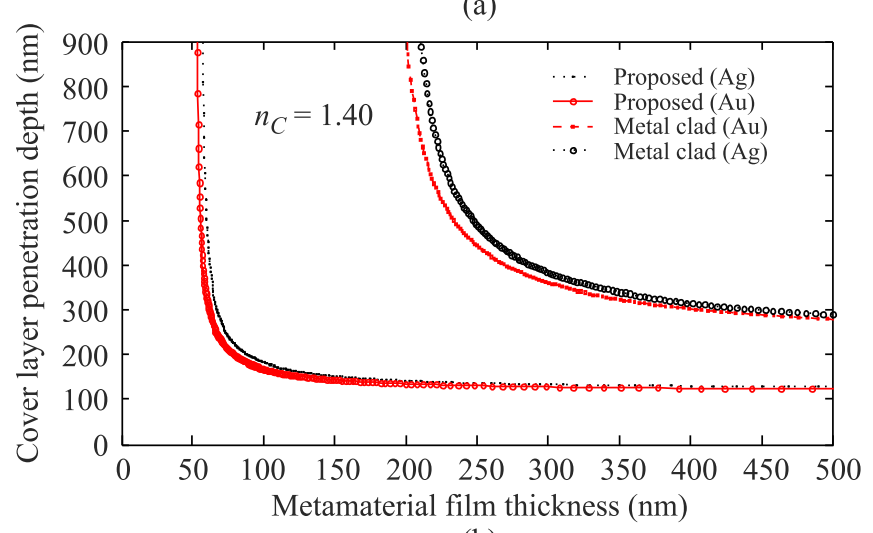

(b)

Fig. 2. Plot of cover layer penetration depth against film thickness for four layer proposed and four layer dielectric sensors in TE mode. The refractive indices used in the simulation are: (a) $n_{S}=1.517, n_{M}=0.065+\mathrm{i} \times 4,0.15+\mathrm{i} \times 3.2, n_{G}=1.59$ and $-\sqrt{[(-4.0+i \times 0.001) \times(-2.4+i \times 0.001)]}, n_{C}=1.33$, (b) $n_{S}=1.517$, $n_{M}=0.065+\mathrm{i} \times 4,0.15+\mathrm{i} \times 3.2, n_{G}=1.59$ and $-\sqrt{[(-4.0+i \times 0.001) \times(-2.4+i \times 0.001)]}, n_{C}=1.40$.

Figure 3(a) reflects change in the cover layer sensitivity $\left(d n_{e f f} d d n_{C}\right)$ against the variation in guiding layer thickness for the fixed value of cover layer refractive index $\left(n_{C}\right)$ equals 1.33. From Fig. 3(a) it is observed that the cover layer sensi-

Table 1. Cover layer penetration depth of the proposed sensor with metamaterial guiding layer, for $n_{S}=1.517, n_{M}=0.065+\mathrm{i} 4(A g)$ and $0.15+\mathrm{i} 3.2(\mathrm{Au}), n_{C}=1.33,1.40$.

\begin{tabular}{|c|c|c|c|}
\hline Type of sensor & Refractive index $\left(n_{C}\right)$ & Metal layer & Cut off thickness (nm) \\
\hline \multirow{4}{*}{ Proposed sensor } & \multirow{2}{*}{1.33} & $\mathrm{Au}$ & 51 \\
\hline & & $\mathrm{Ag}$ & 53 \\
\hline & \multirow{2}{*}{1.40} & $\mathrm{Au}$ & 54 \\
\hline & & $\mathrm{Ag}$ & 56 \\
\hline \multirow{4}{*}{ Metal clad waveguide sensor } & \multirow{2}{*}{$1.33[9]$} & $\mathrm{Au}$ & 160 \\
\hline & & $\mathrm{Ag}$ & 170 \\
\hline & \multirow{2}{*}{1.40} & $\mathrm{Au}$ & 200 \\
\hline & & $\mathrm{Ag}$ & 210 \\
\hline
\end{tabular}




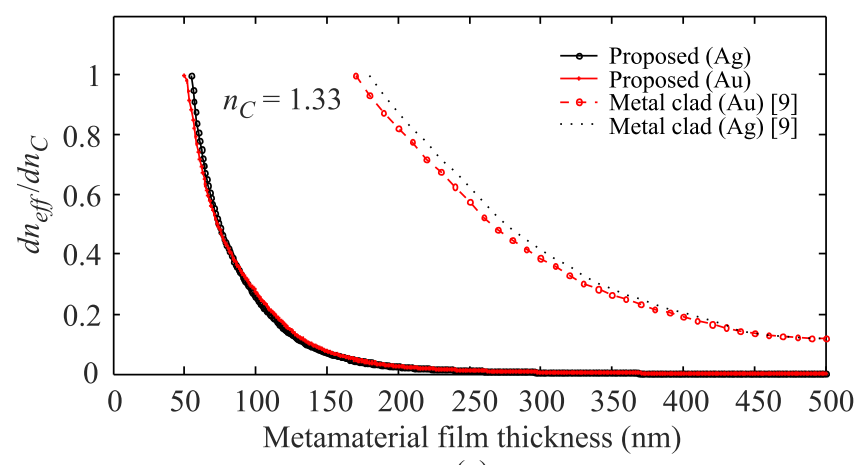

(a)

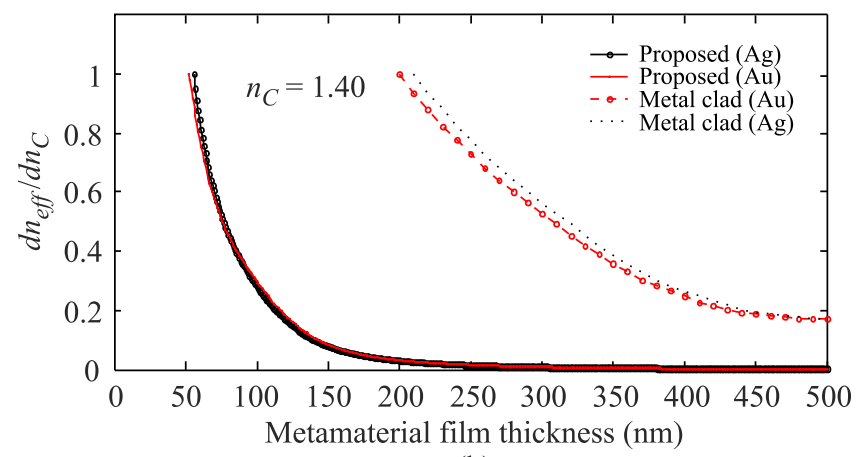

(b)

Fig. 3. Plot of cover layer sensitivity variation versus film thickness for four layer proposed and four layer dielectric sensors in TE mode. The refractive indices used in the simulation are: (a) $n_{S}=1.517$, $n_{M}=0.065+\mathrm{i} * 4,0.15+\mathrm{i} * 3.2, n_{G}=1.59$ and $-\sqrt{[(-4.0+i \times 0.001) \times(-2.4+i \times 0.001)]}, n_{C}=1.33$, (b) $n_{S}=1.517$, $n_{M}=0.065+\mathrm{i} * 4,0.15+\mathrm{i} * 3.2, n_{G}=1.59$ and $-\sqrt{[(-4.0+i \times 0.001) \times(-2.4+i \times 0.001)]}, n_{C}=1.40$.

tivity is 1 at cut off guiding layer thickness, because at cut off thickness value, large fraction of mode power propagates in the cover layer due to infinte penetration depth in the cover layer for the proposed sensor and the metal clad waveguide sensor [9]. Cover layer sensitivity is 1 for the metal clad sensor [9] with $A g$ and $A u$ cladding at $160 \mathrm{~nm}$ and $170 \mathrm{~nm}$ while for the proposed sensor with silver and gold cladding, same cover layer sensitivity value 1 is acheieved at $51 \mathrm{~nm}$ and $53 \mathrm{~nm}$ thickness of guiding layer, respectively. Figures 3(b) also renders change in the cover layer sensitivity $\left(d n_{e f f} / d n_{C}\right)$ against the variation of guiding layer thickness for the fixed value of the cover layer refractive index $\left(n_{C}\right)$ equals 1.40 . Cover layer sensitivity is 1 for the metal clad sensor of silver and gold at $200 \mathrm{~nm}$ and $210 \mathrm{~nm}$ while for the proposed sensor with silver and gold cladding at $53 \mathrm{~nm}$ and $56 \mathrm{~nm}$, respectively. Cover layer sensitivity equals 1 states that the proposed sensor can be efficiently used for a large molecule detection, i.e., in cell detection. Since metamaterial shows lossy nature, hence, the cover layer sensitivity $\left(d n_{e f f} / d n_{C}\right)$ curve of the proposed sensor decreases at faster rate compared to the metal clad sensor [9] by increasing the thickness of a guiding layer. Maximum cover layer sensitivity is achieved at cut off thickness as shown in Table 1.

Figure 4(a) shows variation of the adlayer sensitivity $\left(d n_{e f f} / d d_{A}\right)$ vs. metamaterial film thickness for cover layer refractive index value equals 1.33, at adlayer refractive index equals 1.50. From Fig. 4(a) it is observed that optimum values achieved are $0.661 \times 10^{-3} \mathrm{~nm}^{-1}$ and $0.660 \times 10^{-3} \mathrm{~nm}^{-1}$ at $90 \mathrm{~nm}$ for the proposed sensor with $A g$ and $A u$ cladding, respectively. While the values $0.494 \times 10^{-3} \mathrm{~nm}^{-1}$ and $0.493 \times 10^{-3} \mathrm{~nm}^{-1}$ are achieved at $190 \mathrm{~nm}$ and $192 \mathrm{~nm}$ for dielectric guiding layer thickness with $A g$ and $A u$ cladding, respectively[9]. Fig. 4(b) shows adlayer sensitivity versus metamaterial guiding layer thickness for cover layer refractive index values equals to 1.40 at adlayer refractive index equals to 1.50. From Fig. 4(b), it is seen that optimum values $0.583 \times 10^{-3} \mathrm{~nm}^{-1}$ and $0.53 \times 10^{-3}$ $\mathrm{nm}^{-1}$ are obtained at $95 \mathrm{~nm}$ and $92 \mathrm{~nm}$ for metamaterial guiding layer thickness while the values $0.52 \times 10^{-3} \mathrm{~nm}^{-1}$ and $0.51 \times 10^{-3} \mathrm{~nm}^{-1}$ are obtained at $240 \mathrm{~nm}$ for dielectric guiding layer thickness for the metal clad of $A g$ and $A u$, respectively. Therefore, the adlayer sensitivity $\left(d n_{e f f} / d d_{A}\right)$ is enhanced by using guiding layer of metamaterial, shown in Table 2. Henceforth, the proposed sensor has larger adlayer sensitivity. Thus, the proposed sensor can be efficiently used for a large size

Table 2. Adlayer sensitivity $\left(d n_{e f f} / d d_{A}\right)$ of the proposed sensor with metamaterial as a guiding layer, for $n_{S}=1.517, n_{M}=0.065+\mathrm{i} 4(A g)$ and $0.15+\mathrm{i} 3.2(\mathrm{Au}), n_{C}=1.33 \& 1.40, n_{A}=1.50$ and $\lambda=632.8 \mathrm{~nm}$.

\begin{tabular}{|c|c|c|c|c|}
\hline Type of sensor & Cover layer refractive index $\left(n_{C}\right)$ & Metal layer & $\begin{array}{l}\text { Maximum adlayer sensitivity } \\
\left(10^{-3} \mathrm{~nm}^{-1}\right)\end{array}$ & $\begin{array}{c}\text { Thickness at maximum adlayer } \\
\text { sensitivity (nm) }\end{array}$ \\
\hline \multirow{4}{*}{ Proposed sensor } & \multirow{2}{*}{1.33} & $\mathrm{Au}$ & 0.660 & 90 \\
\hline & & $\mathrm{Ag}$ & 0.661 & 90 \\
\hline & \multirow{2}{*}{1.40} & $\mathrm{Au}$ & 0.530 & 92 \\
\hline & & $\mathrm{Ag}$ & 0.583 & 95 \\
\hline \multirow{4}{*}{ Metal clad } & \multirow{2}{*}{1.33 [9] } & $\mathrm{Au}$ & 0.493 & 190 \\
\hline & & $\mathrm{Ag}$ & 0.494 & 192 \\
\hline & \multirow{2}{*}{1.40} & $\mathrm{Au}$ & 0.510 & 240 \\
\hline & & $\mathrm{Ag}$ & 0.520 & 240 \\
\hline
\end{tabular}




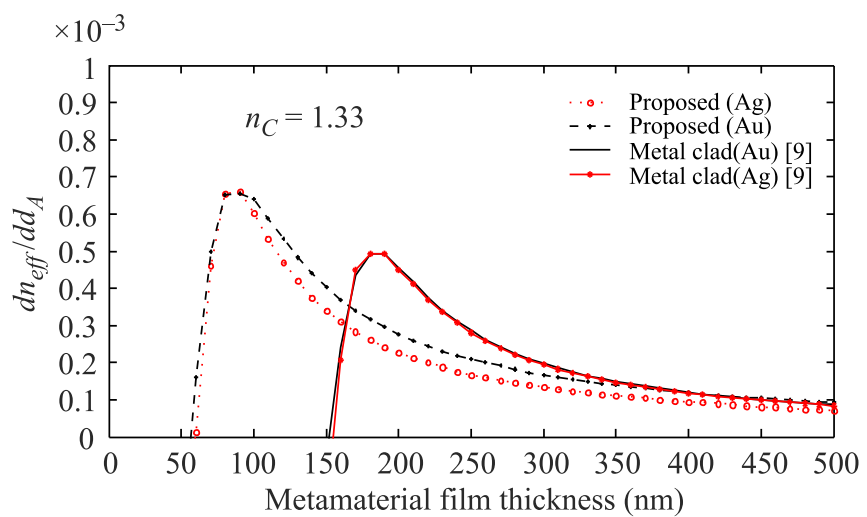

(a)

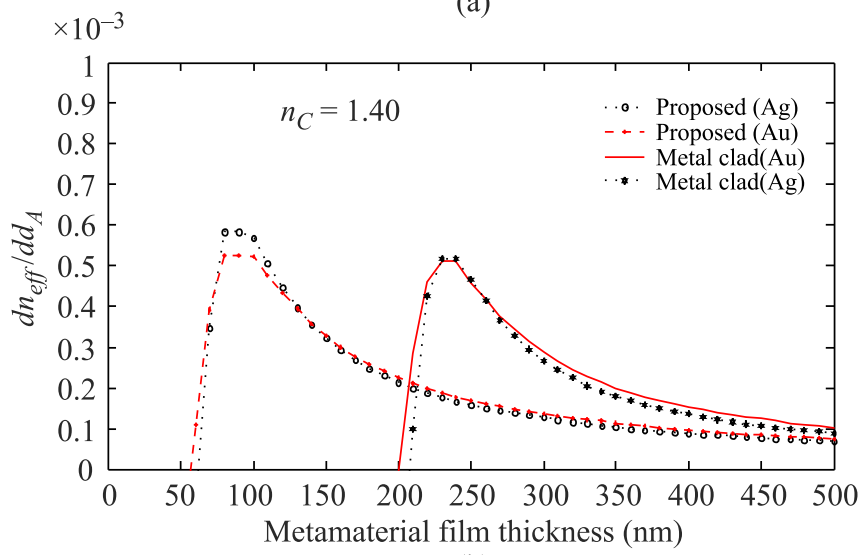

(b)

Fig. 4. (a) Plot of adlayer sensitivity $\left(d n_{e f f} d d_{A}\right)$ versus film thickness for five layer proposed sensor and five layer metal clad waveguide sensor in TE mode. The refractive indices used in the simulation are: $n_{S}=1.517, n_{M}=0.065+\mathrm{i} \times 4,0.15+\mathrm{i} \times 3.2, n_{G}=1.59$ and $-\sqrt{[(-4.0+i \times 0.001) \times(-2.4+i \times 0.001)]}, n_{A}=1.50, n_{C}=1.33,(\mathrm{~b})$ Plot of adlayer sensitivity $\left(d n_{e f f} / d d_{A}\right)$ fluctuation against film thickness for five layer proposed sensor and five layer metal clad waveguide sensor in TE mode. The refractiveindices used in the simulation are: $n_{S}=1.517, n_{M}=0.065+\mathrm{i} \times 4,0.15+\mathrm{i} \times 3.2 n_{G}=1.59$ and $-\sqrt{[(-4.0+i \times 0.001) \times(-2.4+i \times 0.001)]}, n_{A}=1.50, n_{C}=1.40$. biological molecule sensing compared to the metal clad sensor [9].

Figure 5(a) reflects the variation of the adlayer sensitivity $\left(d n_{\text {eff }} d d n_{A}\right)$ at fixed value of 1.33 as the cover layer refractive index $\left(n_{C}\right)$. Optimum values are $0.583 \times 10^{-3} \mathrm{~nm}^{-1}$ at $100 \mathrm{~nm}$ and $0.580 \times 10^{-3} \mathrm{~nm}^{-1}$ at $98 \mathrm{~nm}$ film thickness formetamaterial guiding layer while the values are $0.506 \times 10^{-3} \mathrm{~nm}^{-1}$ at $240 \mathrm{~nm}$ and $0.502 \times 10^{-3} \mathrm{~nm}^{-1}$ at $240 \mathrm{~nm}$ for dielectric guiding layer thickness for $A g$ and $A u$ cladding, respectively at the adlayer refractive index $\left(n_{A}\right)$ value 1.50 . Figure $5(\mathrm{~b})$ presents the variation of adlayer sensitivity at the cover layer refractive index $\left(n_{C}\right)$ value 1.40. Optimum values are $0.573 \times 10^{-3} \mathrm{~nm}^{-1}$ at $101 \mathrm{~nm}$ and $0.580 \times 10^{-3} \mathrm{~nm}^{-1}$ at $100 \mathrm{~nm}$ for metamaterial guiding layer thickness, while the values are $0.508 \times 10^{-3} \mathrm{~nm}^{-1}$ at $240 \mathrm{~nm}$ and $0.492 \times 10^{-3} \mathrm{~nm}^{-1}$ at $240 \mathrm{~nm}$ for dielectric guiding layer thickness with $A g$ and $A u$ cladding, respectively at the adlayer refractive index $\left(n_{A}\right)$ value 1.50 . Adlayer sensitivities, i.e., $\left(d n_{e f f} d d d_{A}\right),\left(d n_{e f f} d d n_{A}\right)$ are zero at cut off film thickness because mode power almost propagates in the cover layer due to an infinte penetration depth of evanescent field in the cover layer, while very small part of mode power propagates in adlayer region adds nothing in adlayer sensitivity. As the guiding layer thickness increases, mode power in the adlayer region also increases due to decrease in mode power in a cover layer. Adlayer sensitivities, i.e., $\left(d n_{e f f} d d d_{A}\right)$ and $\left(d n_{e f f} / d n_{A}\right)$ attain maximum value at the thickness of 40-50 nm larger than the thickness of maximum cover layer sensitivity. Metamaterial guiding layer gives higher sensitivity values in case of the adlayer sensitivity $\left(d n_{e f f} / d d_{A}\right)$ compared to $\left(d n_{e f f} d d n_{A}\right)$, i.e., change due to metamaterial guiding layer is more prominent for the adlayer sensitivity $\left(d n_{e f f} d d d_{A}\right)$. The proposed sensor is exhibiting larger values of adlayer sensitivity in comparison to metal clad sensor [9] for the cover layer refractive index $\left(n_{C}\right)$ values 1.33 and 1.40 as shown in Table 3 . Henceforth, the proposed sensor is better for biosensing application as compared to metal clad waveguide sensor [9].

Table 3. Adlayer sensitivity $\left(d n_{e f f} d d n_{A}\right)$ of the proposed five layer sensor with metamaterial guiding layer for $n_{A}=1.50$. Other parameters are $n_{s}=1.517, n_{M}=0.065+\mathrm{i} 4(\mathrm{Ag})$ and $0.15+\mathrm{i} 3.2(\mathrm{Au}), n_{c}=1.33$ and 1.40 .

\begin{tabular}{|c|c|c|c|c|}
\hline Type of sensor & $\begin{array}{c}\text { Cover layer refractive index } \\
\left(n_{C}\right)\end{array}$ & Metal layer & $\begin{array}{l}\text { Maximum adlayer sensitivity } \\
\left(10^{-3} \mathrm{~nm}^{-1}\right)\end{array}$ & $\begin{array}{c}\text { Thickness at maximum adlayer } \\
\text { sensitivity (nm) }\end{array}$ \\
\hline \multirow{4}{*}{ Proposed sensor } & \multirow{2}{*}{1.33} & $\mathrm{Au}$ & 0.580 & 98 \\
\hline & & $\mathrm{Ag}$ & 0.583 & 100 \\
\hline & \multirow{2}{*}{1.40} & $\mathrm{Au}$ & 0.580 & 100 \\
\hline & & $\mathrm{Ag}$ & 0.573 & 101 \\
\hline \multirow{4}{*}{ Metal clad } & \multirow{2}{*}{1.33} & $\mathrm{Au}$ & 0.502 & 240 \\
\hline & & $\mathrm{Ag}$ & 0.506 & 240 \\
\hline & \multirow{2}{*}{1.40} & $\mathrm{Au}$ & 0.508 & 240 \\
\hline & & $\mathrm{Ag}$ & 0.492 & 240 \\
\hline
\end{tabular}



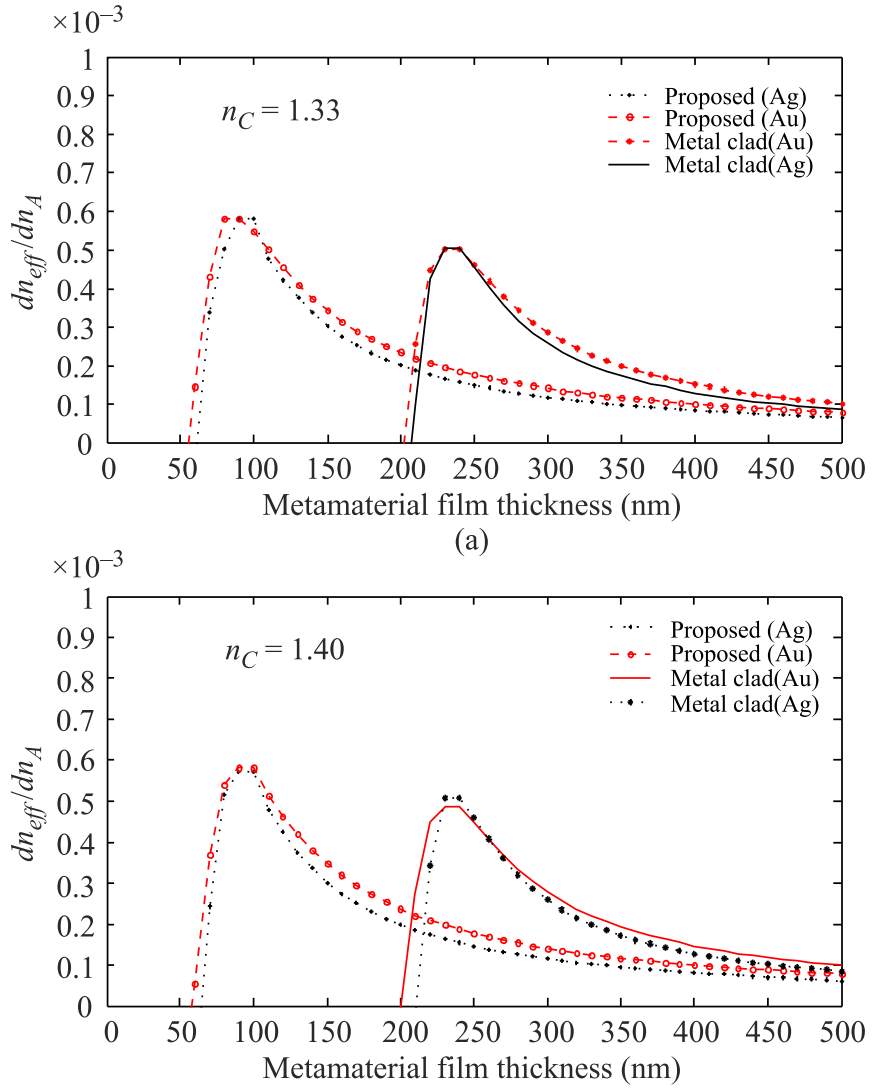

(b)

Fig. 5. Plot of adlayer sensitivity $\left(d n_{e f f} d d n_{A}\right)$ versus film thickness for five layer proposed sensor and five layer metal clad waveguide sensor in TE mode. The refractive indices used in the simulation are: (a) $n_{S}=1.517, n_{M}=0.065+\mathrm{i} \times 4,0.15+\mathrm{i} \times 3.2, n_{G}=1.59$ and $-\sqrt{[(-4.0+i \times 0.001) \times(-2.4+i \times 0.001)]} n_{A}=1.50, n_{C}=1.33$, (b) $n_{S}=1.517, n_{M}=0.065+\mathrm{i} \times 4,0.15+\mathrm{i} \times 3.2, n_{G}=1.59$ and $-\sqrt{[(-4.0+i \times 0.001) \times(-2.4+i \times 0.001)]}, n_{A}=1.50, n_{C}=1.40$.

Figures 6(a) to 9(b) clearly exhibit reflection mode for metal clad [9] and proposed waveguide sensor. The reflectance curve of reflection mode is based on Eq. (8) for TE mode. In the reflection mode, surface plasmon is excited along prism-metal interface and evanescent field is a propagating normal to prism-metal interface. If penetration depth of evanescent field is larger than the aggregate thickness of guiding layer and metal layer, it can be used for sensing applications.

Figures 6(a) and 6(b) represent reflectance curve for the proposed and metal clad waveguide sensor with $A g$ and $A u$ metal cladding, respectively. From Fig. 6(a) it is observed that the curve of the proposed sensor is less sharper. However, change in the resonance angle for the proposed sensor (blue line) is larger than metal clad (black line) [9] which is a deciding factor in order to detect small changes in cover layer refractive index. Hence, change in sensing medium can be easily measured with the proposed sensor. From Fig. 6(b) it is observed that larger shift in resonace angle, as well as larger dip in reflectance curve occur for the proposed sensor (blue line) due to change in cover layer refractive index compared to metal clad (black line) [9]. The refletance curve of the proposed sensor with gold cladding shows lesser sharpness than that of silver cladding. Thus, the proposed sensor has large cover layer sensitivity (change in resonance angle/ change in cover layer refractive index) with $A g$ and $A u$ cladding for TE polarization of input lightas shown in Table 4.

Detection accuracy is defined as $d \theta_{\text {res }} / d \theta_{0.5}$ where $d \theta_{\text {res }}$ denotes the shift in a resonance dip angle due to change in cover layer refractive index, while $d \theta_{0.5}$ represents FWHM (full width half maxima) of the reflectance curve corresponding to 50\% reflectivity [19-20]. The detection accuracy of the proposed sensor is far better than that of metal clad waveguide sensor [9] in case of silver cladding, while it is comparable in case of gold metal cladding as shown in Table 5. However, the sensitivity of the proposed sensor is far better than that of metal clad waveguide sensor [9]. Hence, the proposed sensor is efficient enough to sense even small change in refractive index of cover layer refractive index and also for larger molecule detection in cover layer because large sensitivity corresponds to larger penetration depth in cover layer.

Table 4. Reflectance curve of the proposed four layer sensor with metamaterial guiding filmat parameters $n_{s}=1.517, n_{c}=1.33,1.34$ and 1.35 , $n_{M}=0.065+\mathrm{i} 4(\mathrm{Ag}) \& 0.15+\mathrm{i} 3.2(\mathrm{Au})$.

\begin{tabular}{|c|c|c|c|c|}
\hline Type of sensor & Type of metal cladding & Resonance angle $\left(\theta_{R}\right)$ & Cover layer refractive index $\left(n_{C}\right)$ & Sensitivity $\left(d \theta_{R} / d n_{C}\right)$ \\
\hline \multirow{4}{*}{ Proposed sensor } & \multirow{2}{*}{$\mathrm{Ag}$} & 62.12008 & 1.33 & \multirow{2}{*}{96.83} \\
\hline & & 63.08838 & 1.34 & \\
\hline & \multirow{2}{*}{$\mathrm{Au}$} & 63.25454 & 1.33 & \multirow{2}{*}{115.165} \\
\hline & & 64.40619 & 1.34 & \\
\hline \multirow{4}{*}{$\begin{array}{l}\text { Metal clad waveguide } \\
\text { sensor [9] }\end{array}$} & \multirow{2}{*}{$\mathrm{Ag}$} & 74.30117 & 1.33 & \multirow{2}{*}{21.119} \\
\hline & & 74.51316 & 1.34 & \\
\hline & \multirow{2}{*}{$\mathrm{Au}$} & 74.71943 & 1.33 & \multirow{2}{*}{20.626} \\
\hline & & 74.92569 & 1.34 & \\
\hline
\end{tabular}




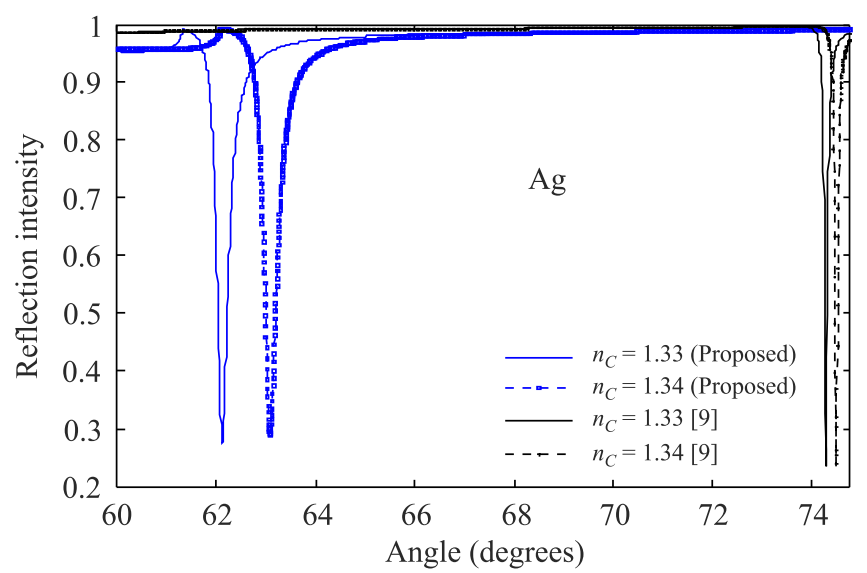

(a)

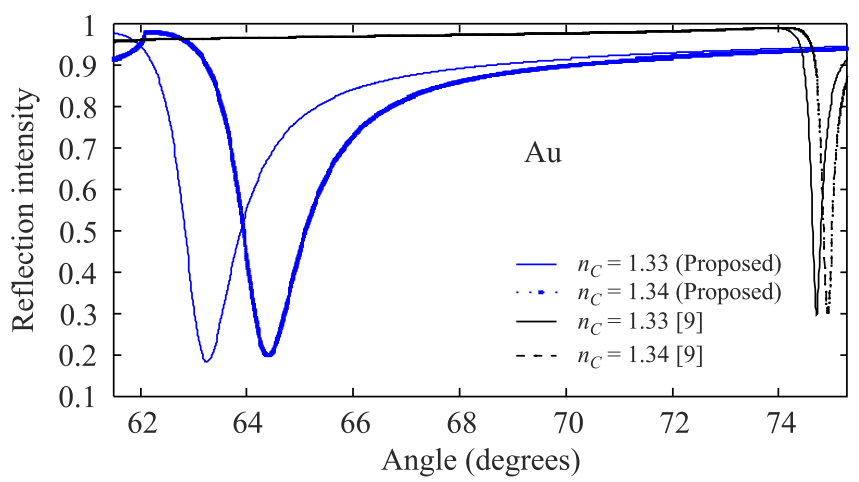

(b)

Fig. 6. (a) Calculated reflectance for TE polarized light reflected from silver clad with dielectric (black line) and metamaterial (blue line) guiding layer for $d_{M}=50 \mathrm{~nm}, d_{G}=350$ and $0 \mathrm{~nm}$, $\lambda=632.8 \mathrm{~nm}, n_{S}=1.517, n_{C}=1.33$ and $1.34, n_{G}=1.59$ and $-\sqrt{[(-4.0+i \times 0.001) \times(-3.0+i \times 0.001)]}, n_{M}=0.065+\mathrm{i} \times 4$, (b) Calculated reflectance for TE polarized light reflected from gold clad with dielectric (black line) and metamaterial (blue line) guiding layer for $d_{M}=50 \mathrm{~nm}, d_{G}=350$ and $0 \mathrm{~nm}, \lambda=632.8 \mathrm{~nm}, n_{S}=1.517, n_{C}=1.33$ and 1.34, $n_{G}=1.59$ and $-\sqrt{[(-4.0+i \times 0.001) \times(-3.0+i \times 0.001)]}$,

$$
n_{M}=0.15+\mathrm{i} \times 3.2 \text {. }
$$

Metal thickness in the proposed sensor has substantial imapact on sharpness and reflectance dip of a reflectance curve. The reflectance dip and sharpness of the calculated reflectance curve are key factors in the sensing application. Figure 7(a) exhibits calculated reflectance curve for different set of values of metal layer thickness for $\mathrm{Ag}$ cladding. Beyond $50 \mathrm{~nm}$, the curve is sharper, but lesser the reflectance minimum, while below $50 \mathrm{~nm}$, the curve has less sharpness but larger the reflectance minimum for the proposed waveguide sensor. Hence, metal thickness value of $50 \mathrm{~nm}$ is an optimum choice of $\mathrm{Ag}$ metal thickness for the proposed sensor. Figure 7(b) shows the reflectance curve for different values of metal layer thickness for $A u$ cladding. Beyond $50 \mathrm{~nm}$, the curve is sharper but lesser the reflectance minimum while below $50 \mathrm{~nm}$, the curve has less sharpness but larger the reflectance minimum for the proposed waveguide sensor. Hence, metal thickness value of $50 \mathrm{~nm}$ is an optimum choice of $A u$ metal thickness for the proposed sensor. It can be concluded that beyond $50 \mathrm{~nm}$ thickness of silver cladding and $60 \mathrm{~nm}$ thickness of gold cladding, the curve does not reflect measurable properties for sensing application. Figures 8(a) and 8(b) are a graph of reflectance intensity variation against the change in incidence angle for metal cladding of $A g$ and $A u$, respectively. Reflectance curve shows a little dependency on guiding layer thickness. On varrying guiding layer thickness parameter, minute shift is introduced in a resonance angle and lower the reflectance minimum of reflectance curve. Decreasing the guiding layer thickness below $350 \mathrm{~nm}$ increases reflectance minimum value but less sharper curve, i.e., increases FWHM value. On the other hand, increase in the guiding layer thickness beyond $350 \mathrm{~nm}$ results into decrease in reflectance minimum value withmore sharper curve, i.e., reduction in FWHM value. Hence, guiding layer thickness of $350 \mathrm{~nm}$ is an optimum choice of guiding layer thickness for both $A g$ and $A u$ metal cladding. Fig. 9(a) and 9(b) exhibit variation in reflectance intensity against incidence angle with change in the adlayer thickness for metal cladding of $A g$ and $A u$, respectively. From figures it is clearly observed that larger shift in

Table 5. Reflectance curve of the proposed four-layer sensor with metamaterial guiding film at parameters $n_{s}=1.517, n_{c}=1.33,1.34$ and 1.35 , $n_{M}=0.065+\mathrm{i} 4(\mathrm{Ag}) \& 0.15+\mathrm{i} 3.2(\mathrm{Au})$.

\begin{tabular}{|c|c|c|c|c|c|}
\hline Type of sensor & $\begin{array}{l}\text { Type of metal } \\
\text { cladding }\end{array}$ & $\begin{array}{c}\text { Resonance angle } \\
\left(\theta_{\text {res }}\right)\end{array}$ & $\begin{array}{c}\text { Cover layer refractive } \\
\text { index }\left(n_{C}\right) \\
\end{array}$ & $\operatorname{FWHM}\left(\theta_{0.5}\right)$ & Detection accuracy \\
\hline \multirow{4}{*}{ Proposed sensor } & \multirow{2}{*}{$\mathrm{Ag}$} & 62.12008 & 1.33 & \multirow{2}{*}{0.18} & \multirow{2}{*}{5.38} \\
\hline & & 63.08838 & 1.34 & & \\
\hline & \multirow{2}{*}{$\mathrm{Au}$} & 63.25454 & 1.33 & \multirow{2}{*}{1.04} & \multirow{2}{*}{1.1} \\
\hline & & 64.40619 & 1.34 & & \\
\hline \multirow{4}{*}{$\begin{array}{l}\text { Metal clad } \\
\text { waveguide sensor [9] }\end{array}$} & \multirow{2}{*}{$\mathrm{Ag}$} & 74.30117 & 1.33 & \multirow{2}{*}{0.16} & \multirow{2}{*}{1.31} \\
\hline & & 74.51316 & 1.34 & & \\
\hline & \multirow{2}{*}{$\mathrm{Au}$} & 74.71943 & 1.33 & \multirow{2}{*}{0.18} & \multirow{2}{*}{1.14} \\
\hline & & 74.92569 & 1.34 & & \\
\hline
\end{tabular}




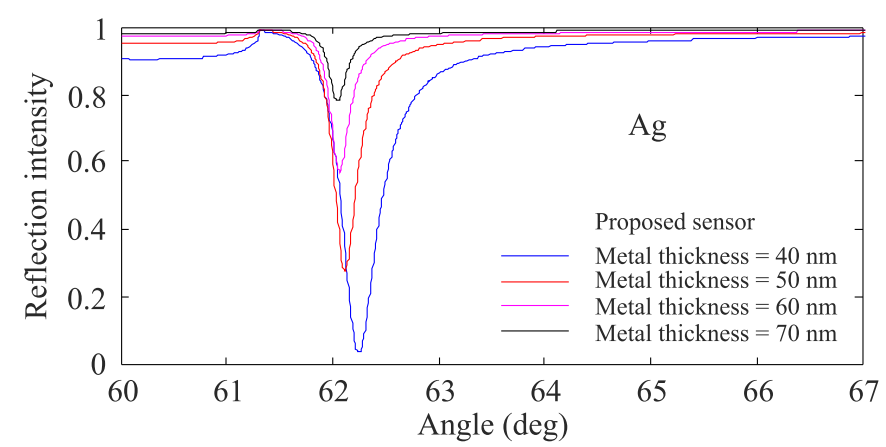

(a)

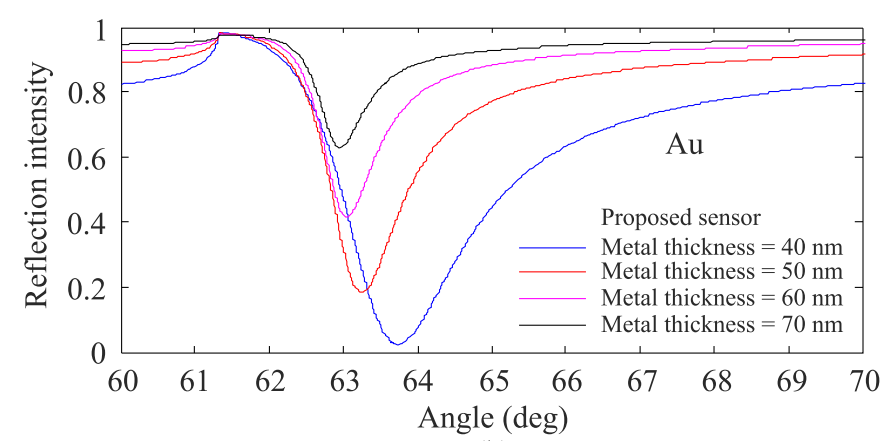

(b)

Fig. 7. (a) Calculated reflectance for TE polarized light reflected from silver clad for different values of metal layer thickness for $d_{G}=350 \mathrm{~nm}, \lambda=632.8 \mathrm{~nm}, n_{C}=1.33, n_{S}=1.517, n_{G}=$ $-\sqrt{[(-4.0+i \times 0.001) \times(-3.0+i \times 0.001)]} n_{M}=0.065+\mathrm{i} \times 4$, (b) Calculated reflectance for TE polarized light reflected from gold clad for different values metal layer thickness for $d_{G}=350 \mathrm{~nm}, \lambda=632.8 \mathrm{~nm}$, $n_{C}=1.33, n_{S}=1.517, n_{G}=-\sqrt{[(-4.0+i \times 0.001) \times(-3.0+i \times 0.001)]}$

$$
n_{M}=0.15+\mathrm{i} \times 3.2 \text {. }
$$

resonance angle occurs due to change in thickness of adlayer for the proposed waveguide sensor in comparison with metal clad waveguide sensor [9] with $A g$ and $A u$ both type of metal claddings. Hence, the adlayer sensitivity (change in resonance angle/change in the thickness of adlayer) is larger for the

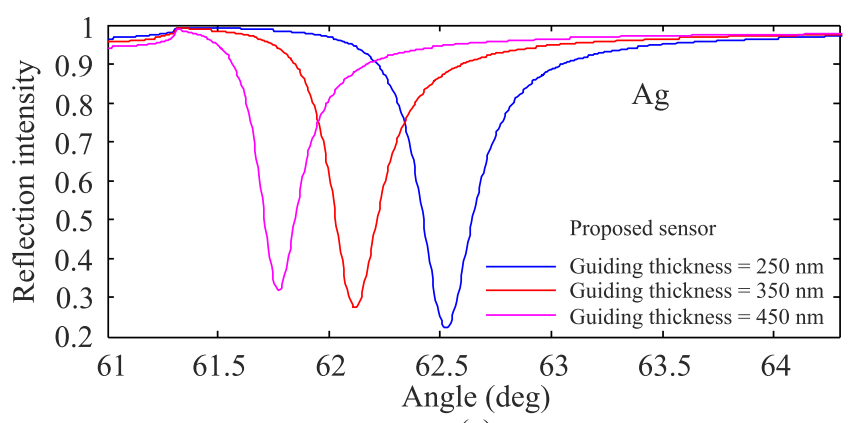

(a)

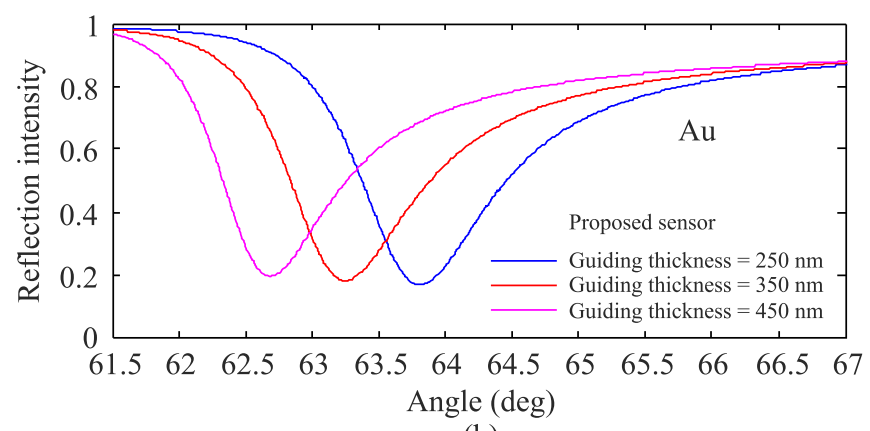

(b)

Fig. 8. (a) Calculated reflectance for TE polarized light reflected from silver clad for different values of guiding layer thickness for $\lambda=632.8 \mathrm{~nm}, n_{S}=1.517, n_{C}=1.33, d_{M}=50 \mathrm{~nm}, n_{G}=$ $-\sqrt{[(-4.0+i \times 0.001) \times(-3.0+i \times 0.001)]} n_{M}=0.065+\mathrm{i} \times 4,(\mathrm{~b})$ Calculated reflectance for TE polarized light reflected from gold clad for different values of guiding layer thickness for $\lambda=632.8 \mathrm{~nm}, n_{S}=1.517, n_{C}=1.33, d_{M}=50 \mathrm{~nm}, n_{G}=$ $-\sqrt{[(-4.0+i \times 0.001) \times(-3.0+i \times 0.001)]} n_{M}=0.15+\mathrm{i} \times 3.2$.

proposed waveguide sensor rather than metal clad waveguide sensor [9] as shown in Table 6.

Apart from this, resolution of the sensor is an important parameter which is defined as the minimum change in the parameter to be determined, which can be differentiated by sensor [21].

Table 6. Reflectance curve of the proposed five layer sensor with metamaterial guiding layer parameters $n_{s}=1.517, n_{c}=1.33$, $n_{M}=0.065+\mathrm{i} 4(\mathrm{Ag}) \& 0.15+\mathrm{i} 3.2(\mathrm{Au}), n_{A}=1.50$.

\begin{tabular}{|c|c|c|c|c|}
\hline Type of sensor & $\begin{array}{l}\text { Type of metal cladding } \\
\left(n_{M}\right)\end{array}$ & $\begin{array}{l}\text { Adlayer thickness }\left(d_{A}\right) \\
(\mathrm{nm})\end{array}$ & Resonance angle $\left(\theta_{R}\right)$ & $\begin{array}{l}\% \text { Adlayer sensitivity } \\
\left(d \theta_{R} / d d_{A} \times 100\right)\end{array}$ \\
\hline \multirow{4}{*}{ Proposed sensor } & \multirow{2}{*}{$\mathrm{Ag}$} & 0 & 62.12008 & \multirow{2}{*}{6.01} \\
\hline & & 2 & 62.24041 & \\
\hline & \multirow{2}{*}{$\mathrm{Au}$} & 0 & 63.25454 & \multirow{2}{*}{10.886} \\
\hline & & 2 & 63.47226 & \\
\hline \multirow{4}{*}{$\begin{array}{l}\text { Metal clad } \\
\text { waveguide sensor [9] }\end{array}$} & \multirow{2}{*}{$\mathrm{Ag}$} & 0 & 74.30117 & \multirow{2}{*}{4.85} \\
\hline & & 2 & 74.39284 & \\
\hline & \multirow{2}{*}{$\mathrm{Au}$} & 0 & 74.71943 & \multirow{2}{*}{4.29} \\
\hline & & 2 & 74.80537 & \\
\hline
\end{tabular}




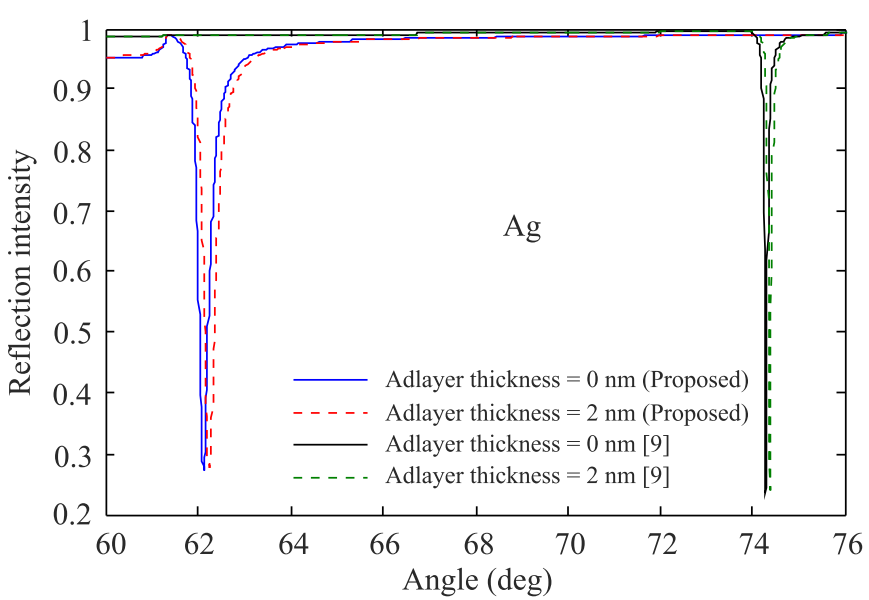

(a)

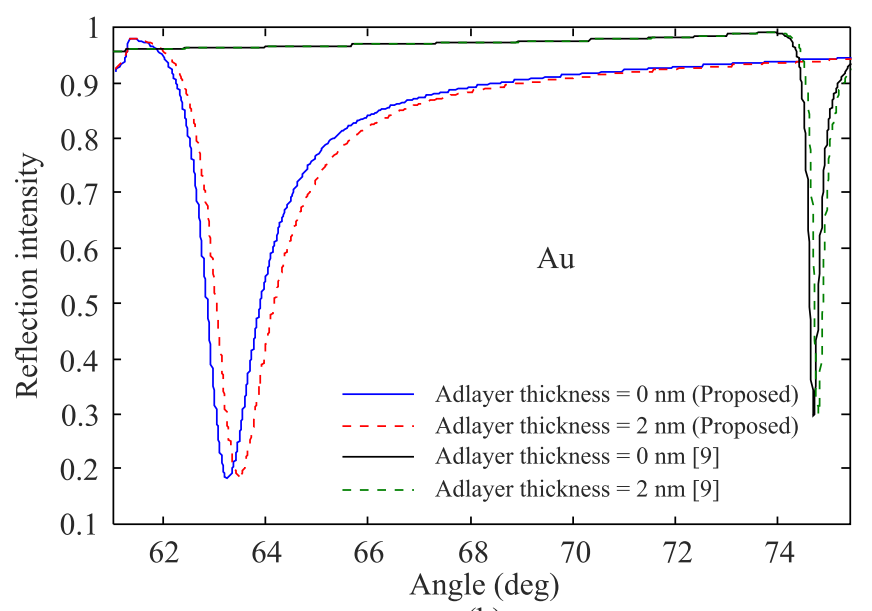

(b)

Fig. 9. (a) Calculated reflectance for TE polarized light reflected from silver clad for different values of adlayer thickness for $d_{G}=350 \mathrm{~nm}$, $\lambda=632.8 \mathrm{~nm}, n_{S}=1.517, n_{C}=1.33, d_{M}=50 \mathrm{~nm}, n_{G}=1.59$ and $-\sqrt{[(-4.0+i \times 0.001) \times(-3.0+i \times 0.001)]}, n_{M}=0.065+\mathrm{i} \times 4, n_{A}=1.50$,

(b) Calculated reflectance for TE polarized light reflected from gold clad for different values of adlayer thickness for $d_{G}=350 \mathrm{~nm}$, $\lambda=632.8 \mathrm{~nm}, n_{S}=1.517, n_{C}=1.33, d_{M}=50 \mathrm{~nm}, n_{G}=1.59$ and $-\sqrt{[(-4.0+i \times 0.001) \times(-3.0+i \times 0.001)]}, n_{M}=0.15+\mathrm{i} \times 3.2$,

$$
n_{A}=1.50 \text {. }
$$

From Fig. 10(a) it is observed that minimum change detected in the cover layer refractive index value of the proposed sensor is 1.33037 and 1.33041 , respectively, while corresponding angle of resonance is 62.11435 and 62.12008 for silver metal cladding. Henceforth, the resolution of the sensor is $4.0 \times 10^{-5}$. From Fig. 10(b) it is observed that minimum change detected in the cover layer refractive index value of the proposed sensor is 1.33038 and 1.33041 , respectively, while corresponding angle of resonance is 63.24881 and 63.25454 for gold metal cladding. Henceforth, the resolution of the sensor is $3.0 \times 10^{-5}$.

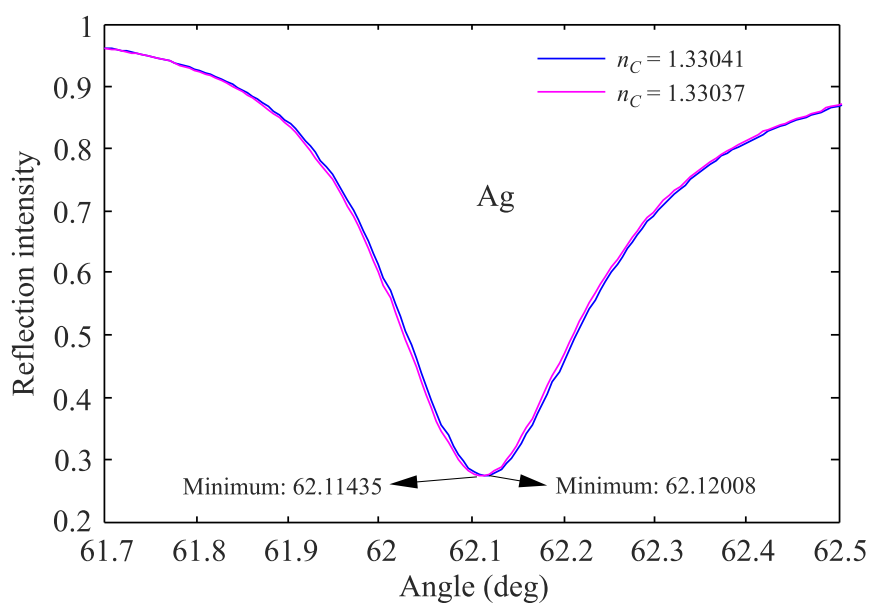

(a)

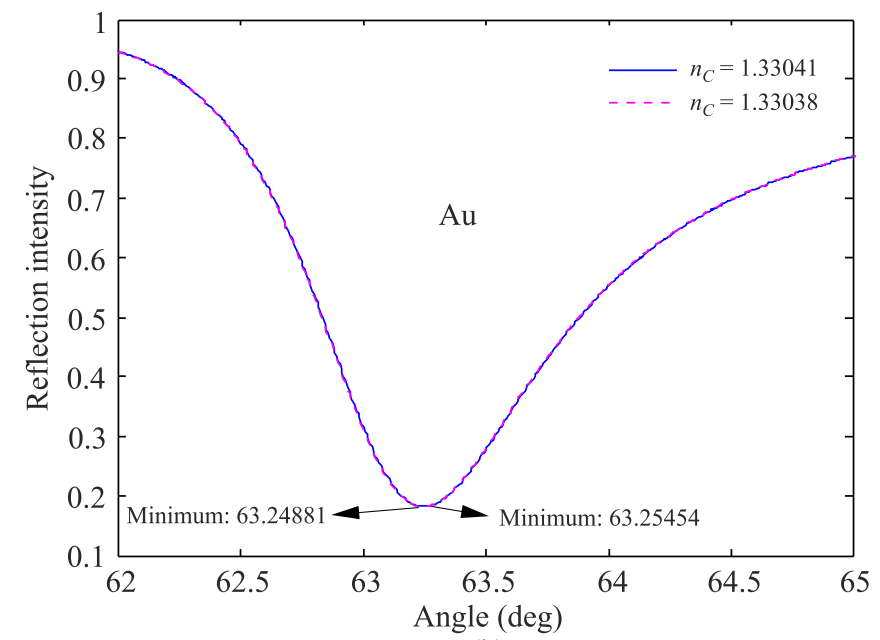

(b)

Fig. 10. (a) Calculated reflectance for TE polarized light reflected from silver clad with metamaterial guiding layer for $d_{M}=50 \mathrm{~nm}$, $d_{G}=350, \lambda=632.8 \mathrm{~nm}, n_{S}=1.517, n_{C}=1.33037$ (mangeta) and 1.33041 (blue), $n_{G}=-\sqrt{[(-4.0+i \times 0.001) \times(-3.0+i \times 0.001)]}$, $n_{M}=0.065+\mathrm{i} \times 4$, (b) Calculated reflectance for TE polarized light reflected from gold clad with metamaterial guiding layer for $d_{M}=50 \mathrm{~nm}$, $d_{G}=350, \lambda=632.8 \mathrm{~nm}, n_{S}=1.517, n_{C}=1.33038$ (mangeta) and 1.33041 (blue), $n_{G}=-\sqrt{[(-4.0+i \times 0.001) \times(-3.0+i \times 0.001)]}$,

$$
n_{M}=0.15+\mathrm{i} \times 3.2 \text {. }
$$

The proposed sensor is applicable in chemical sensing[3], gas and refractive index change measurement [1], biosensing [2]. Apart from this the sensor can also be used in $\mathrm{pH}$ sensing application [22].

\section{Conclusions}

Metal clad waveguide sensor with metamaterial as a guiding layer is analyzed in a waveguiding mode, as well as in reflection mode. The presence of metamaterial guiding layer in the proposed sensor exhibits maximum cover layer sensitivity in a 
waveguiding mode. Large shift in the resonance angle of reflectance curve is obtained for a $350 \mathrm{~nm}$ metamaterial guiding layer between metal layer and cover layer. Henceforth, the proposed sensor exhibits larger cover layer sensitivity than that of metal clad waveguide sensor. Optimum metal thickness is $50 \mathrm{~nm}$ for both type of metal cladding in reflection mode. Adlayer sensitivity is much better for the proposed sensor in comparison to metal clad waveguide sensor. Hence, the proposed sensor shows larger sensitivity for biological sensing. In the reflection mode, $A u$ cladding for proposed sensor exhibits lesser sharpness in the reflectance curve but larger cover layer and adlayer sensitivity. Hence, $A u$ cladding should be preferred over silver cladding for the proposed sensor in sensing application. Hence, using metamaterial as guiding film is beneficial for both, in refractive index measurement and in biosensing. It is clear from discussions that by adjusting the structural parameters, large sensitiviy values might be achieved.

\section{Acknowledgements}

The present work is partially supported by the department of science and Technology (DST), New Delhi, India under the fast track young scientist scheme no. SB/FTP/ETA-0478/ 2012.

\section{References}

1. K. Tiefenthaler and W. Lukosz, "Sensitivity of grating couplers as integrated optical chemical sensors", J. Opt. Soc. Am. B 6 , 209-220 (1989).

2. J. Voros, J.J. Ramsden, G. Csucs, I. Szendro, S.M. dePaul, M. Textor, and N.D. Spencer, "Optical grating coupler biosensors", Biomaterials 23, 3699-3710 (2002).

3. R.E. Kunz, "Miniature integrated optical modules for chemical and biological sensing", Sens. Actuators B 38, 13-28 (1997).

4. W. Lukosz, "Integrated optical chemical and direct biochemical sensors", Sens. Actuators B 29, 3750 (1995).

5. Robert Horvath, H.C. Pedersen, and N. Skivesen, "Monitoring of living cell attachment and spreading using reverse symmetry waveguide sensing", Appl. Phys. Lett. 86, 071101-3 (2005).

6. R. Horvath, L.R. Lindvold, and N.B. Larsen, "Reverse symmetry waveguides: theory and fabrication", Appl. Phys. B. 74, 383-393 (2002).

7. Z. Salamon, S. Cowell, E. Varga, H.I. Yamamura, V.J. Hurby, and G. Tollin, "Plasmon resonance studies of agonist/antagonist binding to the human delta-opioid receptor: New structural insights into receptor-legand interactions", Biophys. J. 79, 2463-2474 (2000).
8. R. Horvath, H.C. Pederson, N. Skivensen, D. Selmeczi, and N.B. Larsen, "Reverse symmetry waveguides: theory and fabrication", Appl. Phys. B. Lett. 86, 383-393 (2005).

9. N. Skivensen, R. Horvath, and H.C. Pedersen, "Optimization of metal clad waveguide sensors", Sens. Actuators B 106, 668-676 (2005).

10. V. Veselago, "The electrodynamics of substance with simultaneously negative values of $\varepsilon$ and $\mu$ ", Sov. Phys. Usp. 10, 509-514 (1968).

11. J.B. Pendry, "Negative refraction makes a perfect lens", Phys. Rev. Lett. 85, 3966-3969 (2000).

12. R.A. Shelby, D.R. Smith, and S. Schultz, "Experimental verification of a negative index of refraction", Science 292, 77-79 (2001).

13. D.K. Qing and G. Chen, "Enhancement of evanescent wave in a waveguide using metamaterials of negative Permittivity and permeability", Appl. Phys. Lett. 84, 669-671 (2004).

14. M. Hujng and J.J. Yang, "Microwave sensor using metamaterial”, A. Petrin Ed., Intech Inc Klagenfurt, pp. 13-36, 2011.

15. K.Y. Yang, V. Giannini, A.O. Bak, H. Amrania, S.A. Maier, and C.C. Phillips, "Subwavelength imaging with quantum metamaterials", Phys. Rev. B 86, 075309 (2012).

16. Y. Prajapati, A. Yadav, A. Verma, V. Singh, and J.P. Saini, "Effect of Metamaterial layer on optical surface plasmon resonance sensor", Int. J. Light Electron Opt. 124, 3607-3610 (2013).

17. S.A. Taya and H.M. Kullab, "Optimization of transverse electric peak type metal clad waveguide sensor using double-negative materials", Appl. Phys. A 116, 1841-1846 (2014).

18. D. Sharma, A. Verma, Y.K. Prajapati, V. Singh, and J.P. Saini, "Forward and backward wave propagation in multilayer planar waveguide using metamaterials layer", Opt. Quant. Electron 45, 105-114 (2013).

19. J.B. Maurya, Y.K. Prajapati, V. Singh, and J.P. Saini, "Sensitivity enhancement of surface plasmon resonance sensor based on graphene-MoS2 hybrid structure with TiO2-SiO2 composite layer", Appl. Phys. A-Materials Science \& Processing 121, 525-533, DOI 10.1007/s00339-015-9442-3, (2015).

20. J.B. Maurya, Y.K. Prajapati, V. Singh, J.P. Saini, and R. Tripathi, "Performance of graphene-MoS2 based surface plasmon resonance sensor using silicon layer," J. Opt. and Quant. Electron. 47, 3599-3611 (2015).

21. R. Kashyap and G. Nemova, "Surface plasmon resonance-based fiber and planarwaveguide sensors", J. Sens. DOI: 10.1155/2009/645162, (2009).

22. O. Korostynska, K. Arshak, E. Gill, and A. Arshak, "Review on state-of-the-art in polymer based ph sensors", Sensors 7, 3027-3042 (2007).

23. A. Upadhyay, Y.K. Prajapati, V. Singh, and J.P. Saini, "Sensitivity estimation of metamaterial loaded planer waveguide sensor", Opt. Quant. Electron 47, 2277-2287 (2015). 